\title{
Electrodynamic Tether for Scientific Mission in Low Jovian Orbit
}

\author{
Juan R. Sanmartin, ${ }^{*}$ Claudio Bombardelli, ${ }^{\dagger}$ Mario Charro $^{\ddagger}$ and Enrico Lorenzini ${ }^{\S}$
}

\begin{abstract}
An electrodynamic bare tether is shown to allow carrying out scientific observations very close to Jupiter, for exploration of its surface and subsurface, and ionospheric and atmospheric in-situ measurements. Starting at a circular equatorial orbit of radius about 1.3/1.4 times the Jovian radius, continuous propellantless Lorentz drag on a thin-tape tether in the 1-5 km length range would make a spacecraft many times as heavy as the tape slowly spiral in, over a period of many months, while generating power at a load plugged in the tether circuit for powering instruments in science data acquisition and transmission. Lying under the Jovian radiation belts, the tape would avoid the most severe problem facing tethers in Jupiter, which are capable of producing both power and propulsion but, operating slowly, could otherwise accumulate too high a radiation dose . The tether would be made to spin in its orbit to keep taut; how to balance the Lorentz torque is discussed. Constraints on heating and bowing are also discussed, comparing conditions for prograde versus retrograde orbits. The system adapts well to the moderate changes in plasma density and motional electric field through the limited radial range in their steep gradients near Jupiter.
\end{abstract}

\section{Introduction}

Power and propulsion needs have been permanent critical issues in missions to the outer planets. All past missions to Jupiter, in particular, powered onboard instruments by using Radioisotope Thermal (or Power) Generators (RTGs), which present, however, a number of problems. Furthermore, a very recent Report from its National Research Council warned that the US was running out of RTGs fuel (Pu-238); production was stopped about 20 years ago, though NASA keeps buying some from Russia and using about $5 \mathrm{~kg}$ each year [1]. This has brought to being, in the coming Jovian mission Juno, a poor, heretofore ignored alternative, which is use of the weak solar power reaching Jupiter. Also, the two-spacecraft Europa Jupiter System Mission (EJSM), jointly planned by NASA and ESA for 2020, will use RTGs in NASA's Jupiter Europa Orbiter (JEO) but solar panels in ESA's Jupiter Ganymede Orbiter (JGO).

In turn, capture into and touring in the deep Jovian gravitational well typically require a very high wet-mass fraction if chemical rockets (in addition to gravity assist operations) are used for propulsion, as was always the case in the past. In particular, the JEO system mass budget is made of $2646 \mathrm{~kg}$ of Propellant, $1367 \mathrm{~kg}$ of Flight System Mass, and $123 \mathrm{~kg}$ of Launch Vehicle Adapter, with $973 \mathrm{~kg}$ left for

\footnotetext{
${ }^{*}$ Universidad Politécnica de Madrid (UPM), School of Aeronautics, Madrid, E-28040, Spain.

${ }^{\dagger}$ Universidad Politécnica de Madrid (UPM), School of Aeronautics, Madrid, E-28040, Spain.

¥Universidad Politécnica de Madrid (UPM), School of Aeronautics, Madrid, E-28040, Spain.

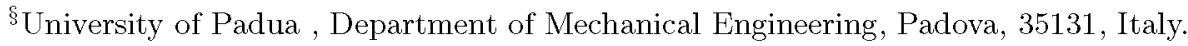


contingency and margin, for a Launch Mass Capability of $5040 \mathrm{~kg}$. Corresponding values for the JGO are $2562,957,190,653$, and $4362 \mathrm{~kg}$, respectively. Both JEO and JGO will use ballistic trajectories with gravity assists from Venus and Earth [2].

Beyond a range of other applications [3], Bare Electrodynamic Tethers can provide both power and propulsion, with just tether hardware accounting for the tether subsystem mass. Basically, a planetary magnetic field induces a motional electric field $\mathbf{E}_{\mathbf{m}}$ in the orbiting-tether frame, and exerts a Lorentz force on the current that $\mathbf{E}_{\mathbf{m}}$ drives through the tether. If used at Jupiter, tethers with a moderate tether-hardware/spacecraft mass ratio have been proved able to perform Jovian Orbit Insertion followed by a moons tour with near zero wet mass, while providing power throughout the entire operation [4], [5]. A total of 40 flybys of Ganymede, Europa and Io (as against 25 moon flybys planned for $\mathbf{J E O}$ ), could be carried out before some limiting radiation dose did accumulate.

Radiation dose is the main constraint on tether use at Jupiter because operation is requiring on ambient conditions, i.e. on plasma density and magnetic field, so orbits must reach near Jupiter (at the Io torus, density is as high as near Jupiter, but the magnetic field appears too weak). For missions such that orbits skip the Jovian Radiation Belts, anyway, tethers would be greatly useful. The Juno case is a particular example. It was recently shown that a moderately light tether might provide the power its SC requires [6].

In this work we consider a mission final-stage with the SC starting in circular, equatorial orbit at a radius $a_{i}$ about 1.3-1.4 times the Jovian radius $r_{J}$. It has been recently shown how to get the $\mathrm{SC}$ to such initial condition [7]; after the capture described in [4], the SC tether would have its apoapsis progressively lowered to finally reach a circular orbit with radius the perifocus of the capture orbit, skipping the moon flybys considered in [4]so as to reduce the accumulated radiation dose. Afterwards, the tether can make its SC spiral in slowly, in a controlled manner, keeping below the Belts throughout while generating power onboard. The proximity to Jupiter, the in situ conditions, first at a particulate ambient and later down in the atmosphere, and the possible use of the tether itself as instrument in active experiments, offer a world of scientific opportunities, which are discussed in the following section.

\section{Mission Science Capabilities}

The radial range 1.3-1.4 $r_{J}$ contains both the inner region of the Halo ring and the 2:1 Lorentz resonance $\left(\Omega_{o r b}=2 \Omega_{J}\right)$. The Lorentz force on a charged grain results in sensible grain acceleration because of large Jovian magnetic field and orbital velocities, and charge-to-mass ratios (Jovian grains have $0.1-1 \mu \mathrm{m}$ size and get charged to - $10 \mathrm{~V}$ ). Lorentz resonances occur at circular, equatorial orbits that are commensurate with some periodicity in the magnetic field, arising in its multipole expansion, from terms beyond the tilted, offset dipole. The 2:1 Lorentz resonance, basically due to a Schmidt coefficient $\left|g_{2}^{2}\right| \sim 0.4-0.5$ Gauss, is the strongest by far and is located at about $1.4 r_{J}$. In situ detection of (charged) grains might advance well beyond remote-imaging ring studies [8].

A basic scientific goal relates to the determination of the structure and dynamics of the Jovian atmosphere, which is actually one goal of the JESM too. Spatially resolved observations, essential for the understanding of transport processes, will be possible. Data registered over many months would allow studying atmospheric variability over different time scales. This includes clarifying how and why the stratospheric thermal structure varies with time, and tracking the evolution of lightning storms[9].

Measurements so close to Jupiter's surface would also allow increasing our knowledge of its interior. This would include accurate, high resolution determination of the gravity and magnetic fields, and determining the bulk abundance of water. Accurate mapping of the gravity field could show non- 
hydrostatic pressure effects where water was absent. Measuring brightness temperature at millimeter wavelengths at close range would give water abundance without the ambiguity of remote sensing [9].

Beyond passive measurements, the orbiting bare tether would allow some active experiments. As explained in Sec. 4 , as the tether rotates at high spin to keep taut, it will be electrically floating half of the time, with current vanishing at both anodic and cathodic ends. Because of the large ion-toelectron mass ratio, the field $\mathbf{E}_{\mathbf{m}}$ will have the tether biased negative over most of its length. Under the impact of attracted ions, the tether will emit secondary electrons, which form a beam travelling along magnetic field lines. For the multipole Jovian magnetic field, the beam ejected from equatorial orbit could magnetically mirror back and forth but also imprint auroral lights at the lower atmosphere, along a beam track [10].

\section{Low Jupiter Orbit Environment}

Low altitude Equatorial orbits around Jupiter are ideal for the operation of electrodynamic tethers. Radiations are virtually zero for orbital radii not exceeding 1.3-1.4 Jupiter radii, i.e. below the inner trapped radiation belt. In addition, a relatively strong magnetic field and dense atmosphere are available, which boosts the electrodynamic tether performance.

Jupiter plasmasphere can be assumed corotating with Jupiter at $\Omega_{J}=1.77 \times 10^{-4} \mathrm{rad} / \mathrm{s}$ with local electron density $N_{e}$ provided by the Divine-Garrett model [11]. Dominant ion species are sulphur and oxygen. For the purpose of the present analysis, longitude-related density variations can be neglected and the orbit can be assumed virtually circular thruought its evolution providing the simple expression for the electron density:

$$
N_{e}=N_{0} \exp \left(\frac{r_{0}}{a}\right) .
$$

A zero-offset tilted dipole model for the magnetic field will do for our analysis. Since the tether is in equatorial orbit with its attitude confined to the orbital plane the relevant magnetic field component is along Jupiter spin axis:

$$
B=\frac{B_{S} r_{J}^{3}}{a^{3}} \cos \beta,
$$

where $B_{S}$ is the planetary surface magnetic field at zero latitude and $\beta$ is the magnetic field tilt angle which can here be taken as $\beta \sim 9.6 \mathrm{deg}$.

\section{Power Generation vs Orbit Decay}

Let us consider a bare electrodynamic tether initially in a generic equatorial orbit around Jupiter and spinning around an axis orthogonal to the orbit plane. Given the strong magnetic and gravitational field in low Jupiter orbit and the relatively low plasma density, when compared to Earth, ohmic effects can be safely neglected even for very thin tethers of length up to several kilometers. In addition, given the limited size of the tether cross section, in our case a tape of width w and thickness $\mathrm{h}$, charge collection occurs under orbital motion limited (OML) regime.

The current averaged over both the tether line and the spin period can be readily computed from reference [6] with the addition of the effect of ion collection. Since both tether line and motional electric field vector lie in the orbital plane at angle $\varphi$ we have: 


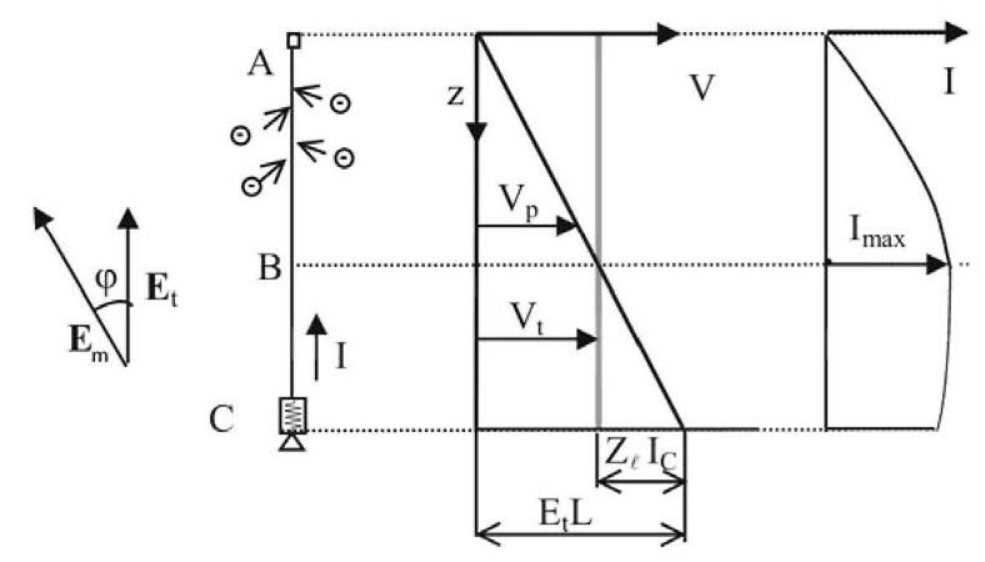

Figure 1: Schematics of bare EDT working in generator mode with negligible ohmic losses along the tether. $V_{p}$ and $V_{t}$ indicate, respectively, the plasma and tether potential. Points $\mathrm{A}, \mathrm{B}$ and C indicate anodic end, zero bias point and cathodic end. $I$ is the conventional (positive) current. $E_{t}$ is the component of the motional electric field $\mathbf{E}_{m}$ along the tether line.

$$
\begin{aligned}
\overline{I_{a v}} & =I_{a v}(\varphi=0) \times \overline{\sqrt{\cos \varphi}}= \\
& =\overline{\sqrt{\cos \varphi}} \frac{\zeta^{3 / 2}(5-2 \zeta)-2 \mu(1-\zeta)^{3 / 2}}{3} \times \frac{2}{5} q_{e} N_{e} \times \frac{2 w L}{\pi} \sqrt{\frac{2 q_{e} E_{m} L}{m_{e}}},
\end{aligned}
$$

where $I_{a v}(\varphi)$ is the current averaged along the tether line, $L$ the tether length $\zeta L$ the length of the anodic tether segment, $w$ the tape width, $E_{m}$ the local motional electric field, $N_{e}$ the electron density of the local ambient plasma, $q_{e}$ and $m_{e}$ electron charge and mass and the parameter $\mu=\sqrt{m_{e} / m_{i}}$ the square root of the electron to ion mass ratio in Jupiter ionosphere $\left(\mu \sim 5 \times 10^{-3}\right)$. Finally, $\overline{\sqrt{\cos \varphi}}$ is a reduction coefficient which takes into account tether rotation. In the present design a cathodic contactor is active along only half a rotation of the tether, which is left floating during the other half. In this way, and neglecting the small current collected by the floating tether, we have:

$$
\overline{\sqrt{\cos \varphi}}=\frac{1}{2 \pi} \int_{-\pi / 2}^{\pi / 2}(\cos \varphi)^{1 / 2} d \varphi \cong 0.381 .
$$

The resulting rotation-averaged Lorentz force has magnitude:

$$
\begin{aligned}
|\overline{\mathbf{F}(\varphi)}| & =|\mathbf{F}(\varphi=0)| \times \overline{(\cos \varphi)^{3 / 2}}= \\
& =\overline{\overline{(\cos \varphi)^{3 / 2}}} \frac{\zeta^{3 / 2}(5-2 \zeta)-2 \mu(1-\zeta)^{3 / 2}}{3} \times \frac{2}{5} q_{e} N_{e} B E_{m} \times \frac{2 w L}{\pi} \sqrt{\frac{2 q_{e} E_{m} L}{m_{e}}}
\end{aligned}
$$

where the rotation reduction coefficient is in this case:

$$
\overline{(\cos \varphi)^{3 / 2}}=\frac{1}{2 \pi} \int_{-\pi / 2}^{\pi / 2}(\cos \varphi)^{3 / 2} d \varphi \cong 0.278
$$

Finally the power generated through a load placed right before the plasma contactor at the cathodic end of the tether obeys: 


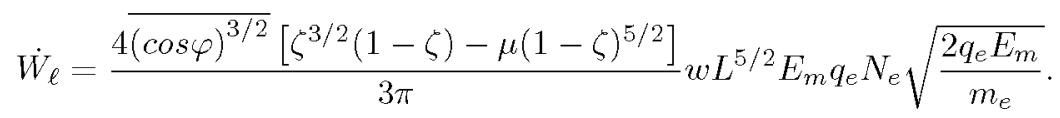

Note that there are two values of the parameter $\zeta$ yielding zero power at the load:

$$
\begin{aligned}
& \tilde{\zeta}_{1}=1, \\
& \tilde{\zeta}_{2}=\frac{\mu^{2 / 3}}{\mu^{2 / 3}+1} \cong \mu^{2 / 3},
\end{aligned}
$$

the first value corresponding to zero load (maximum current) while the second providing the floating tether condition (zero current at the cathode).

Assuming an initially circular orbit and neglecting eccentricity variations throughout the spiral-in phase the motional electric field $\mathbf{E}_{m}$ is radial and has magnitude:

$$
E_{m}=\left(\Omega_{o r b} \pm \Omega_{J}\right) a B
$$

where $\Omega_{\text {orb }}$ is the orbital angular velocity, $a$ is the orbit radius and the - $(+)$ sign refers to a prograde (retrograde) orbit.

The time evolution of the orbit radius is described by a Lagrange planetary equation in the Gauss form:

$$
\frac{d a}{d t}=2 a^{2} \frac{v_{s c}}{\mu_{J}} \frac{F}{m_{s c}},
$$

where $v_{s c}, m_{s c}$ are spacecraft velocity and total mass while $\mu_{J}$ is the Jupiter gravitational constant.

At this point we consider the parameter:

$$
\varepsilon=-\frac{1}{m_{s c}} \frac{d W}{d a}=-\frac{\dot{W}}{m_{s c}} \frac{d t}{d a},
$$

which describes the specific energy extracted from the spacecraft and plasma kinetic energy per unit of orbit radius variation and spacecraft mass. By using Eqs. $(7,11)$ in Eq. (12), straightforward algebra yields:

$$
\varepsilon=-\frac{5 \mu_{J}}{2 a^{2}} \frac{\zeta^{3 / 2}(1-\zeta)-\mu(1-\zeta)^{5 / 2}}{\zeta^{3 / 2}(5-2 \zeta)-2 \mu(1-\zeta)^{3 / 2}}\left(1 \pm \frac{\Omega_{J}}{\Omega_{\text {orb }}}\right) .
$$

Eq.(13) shows that a retrograde orbit allows to extract, for the same orbit decay, almost twice the amount of energy that would be obtained with a prograde orbit, as $\Omega_{J} / \Omega_{\text {orb }} \sim 0.3$ for an average value of $a=1.15 r_{J}$. Furthermore, an additional gain in energy extracted can be obtained by reducing the control parameter $\zeta$ (by increasing the impedance of the load). On the other hand moving $\zeta$ away from its optimum value $(3 / 5)$ will reduce the specific power extracted per unit of tether mass.

Assuming $\zeta$ constant during the spiral-in phase the total energy extracted in going from a radius $a_{\text {out }}$ to $a_{\text {in }}$ is:

$$
\begin{gathered}
\dot{W}_{\ell}=m_{s c} \int_{a_{\text {oiu }}}^{a_{\text {in }}} \varepsilon d a=\frac{5}{2} m_{s c} \sqrt{\mu_{J}} \frac{\zeta^{3 / 2}(1-\zeta)-\mu(1-\zeta)^{5 / 2}}{\zeta^{3 / 2}(5-2 \zeta)-2 \mu(1-\zeta)^{3 / 2}} \times \\
{\left[\sqrt{\mu_{J}}\left(\frac{1}{a_{\text {in }}}-\frac{1}{a_{\text {out }}}\right) \pm 2 \Omega_{J}\left(\sqrt{a_{\text {out }}}-\sqrt{a_{\text {in }}}\right)\right]}
\end{gathered}
$$



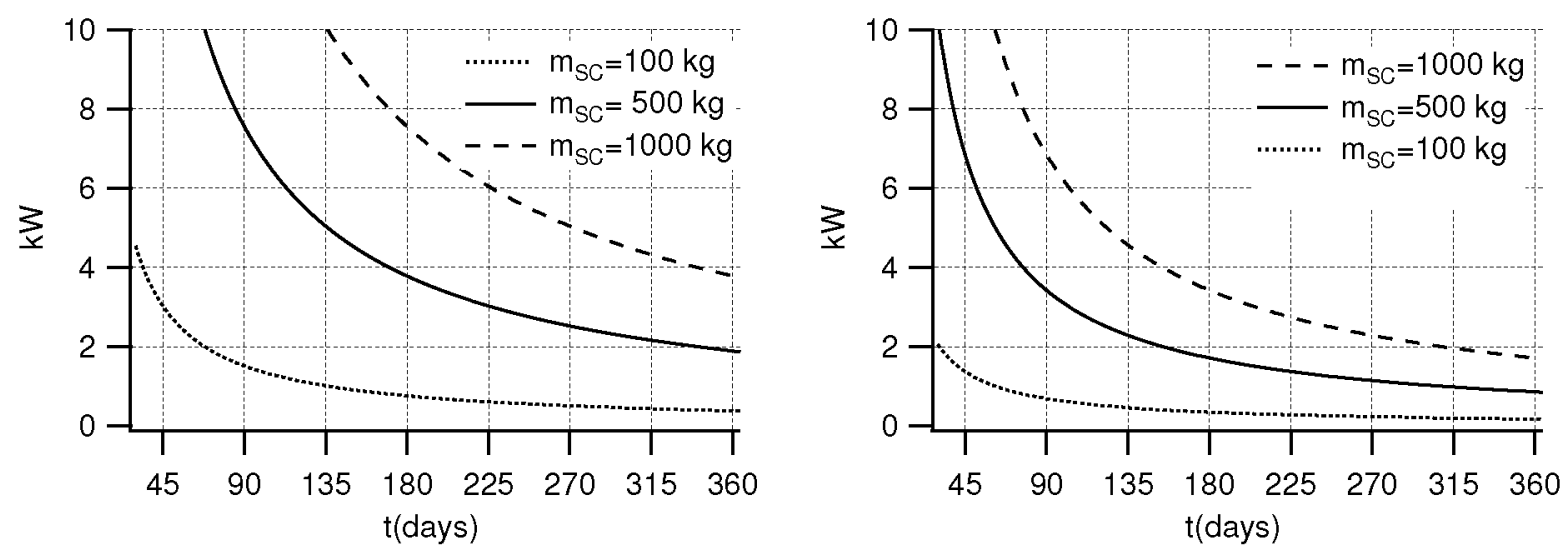

Figure 2: Average power generated during deorbiting from 1.3 to $1.05 r_{J}$ orbital radius for a given mission duration $(t)$ and spacecraft mass $\left(m_{S C}\right)$ and considering a circular retrograde (a) and prograde (b) orbit. The parameter $\zeta$ is fixed at 0.6 .

which can be used to estimate the average power for a given mission time and spacecraft mass as described in Figure (2).

\section{Tether Design and Performance}

One important driver for the tether design comes from the need to reach a low altitude orbit around Jupiter with a payload of reasonable mass. The maneuver $\Delta V$ needed to circularize an orbit with periapsis radius $r_{p}$ and eccentricity $e$ is:

$$
\Delta V=\sqrt{\frac{\mu_{J}}{r_{p}}}(\sqrt{1+e}-1)
$$

For example considering $r_{p}=1.3 r_{J}$ and an initial parabolic orbit $(\mathrm{e}=1)$ we obtain a $\Delta V$ of more than $15 \mathrm{~km} / \mathrm{s}$. While such a maneuver is clearly far beyond the reach of current propellant-based propulsion systems an electrodynamic tether of about $50 \mathrm{~km}$ has been proved capable of progressively lowering the apojove to reach a low Jovian circular orbit in about 18 perijove passes, for a tether mass fraction $\alpha=m_{t} / m_{S C}$ about $1 / 4$. The same tether would then be employed for the present mission concept after trimming the produced power output by reducing its length (e.g. by cutting or retrieving part of the tape), by increasing the load impedance thus shortening the length of the anodic section $(\zeta)$ or by switching off the hollow cathode over part of its negative bias half-period.

Once the geometric characteristics of the tether are set the spacecraft motion and generated power can be computed according to Eqs. $(7,11)$ after considering the dependency of the magnetic field intensity and plasma density with the spacecraft position. After substituting Eqs.(1,2) into Eq.(5) and solving Eq.(11) we obtain the evolution of the orbit radius $a$, and the generated power profile, which is plotted in Fig. (3) for different value of tether length fixed value $\zeta=0.6$.

The point of application of the Lorentz force lies at a distance from the anodic end [6]:

$$
d=\frac{L}{14} \frac{8 \zeta^{7 / 2}-35 \zeta^{3 / 2}+4 \mu(1-\zeta)^{5 / 2}(5+2 \zeta)}{2 \zeta^{5 / 2}-5 \zeta^{3 / 2}+2 \mu(1-\zeta)^{5 / 2}} .
$$

In order to have constantly zero torque the overall tether center of mass will be constrained to lay at the same distance from the anodic end. Note, however, that the value of $d$ can be tuned with the control parameter $\zeta$ so that the zero torque condition can be met even in the case of off-nominal 


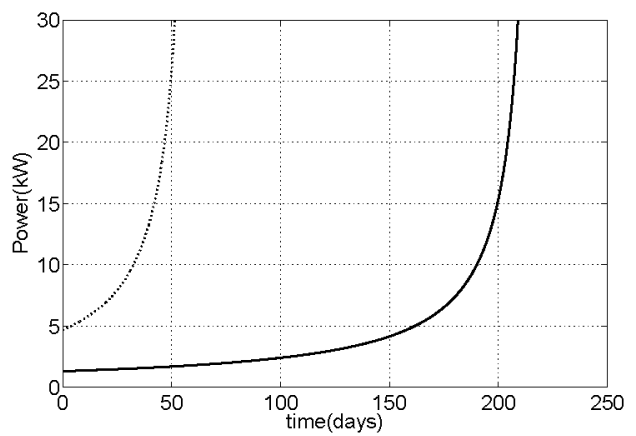

Figure 3: Rotation-averaged power generated during deorbiting from 1.4 to $1.05 r_{J}$ orbital radius for spacecraft of $500 \mathrm{~kg}$ mass and equipped with an electrodynamic tether of length $5 \mathrm{~km}$ (dash line) and $3 \mathrm{~km}$ (solid line). Tether width is $5 \mathrm{~cm}$ while the parameter $\zeta$ is kept constant to the value of 0.6 .

conditions for center of mass location. For the same reason, employing a variable $\zeta$ along the mission (e.g. for power control purposes) would generally introduce a non-zero torque which will tend to spin up or down the tether. On the other hand, since the power can be reduced by both increasing and decreasing $\zeta$ from the optimum value $\left(\zeta_{\text {opt }} \simeq 3 / 5\right)$ it is generally possible to alternate spin-up and spin-down maneuver events in such a way as to balance the overall angular rate variation along a given period of time hence eliminating secular variations of angular velocity.

As far as tether survivability against micrometeoroids is concerned, the choice of a tape cross section helps keeping the probability of having the tether severed to an extremely low value. For instance, dust grains accumulated around the 2:1 Lorentz resonance radius, most critical for the tether, are typically many orders of magnitude smaller than tape widths considered for this application.

\section{Tether Heating and Bowing}

In the Jupiter environment, where solar irradiation is small and ohmic effects are negligible for practicably sized tethers, heating is primarily due to the impact of electrons collected at bias $\Delta V(x)$ along the tether. The energy balance can be written as [4]:

$$
\frac{\partial T}{\partial t}=\frac{\dot{w_{t h}}}{w h \rho_{t} c_{t}}-\frac{2 \varepsilon_{t} \sigma_{B} T^{4}}{h \rho_{t} c_{t}},
$$

where $\rho_{t}, c_{t}, \varepsilon_{t}$ and $h$ are, respectively, the tether material density, specific heat, emissivity and tape thickness, $\sigma_{B}$ is the Stefan-Boltzmann constant, $T$ is tether temperature and $\dot{w}_{t h}$ the local heating power per tether unit length due to electron impact:

$$
\dot{w}_{t h}=\Delta V \frac{d I}{d x}=\frac{2 w}{\pi} q_{e} N_{e} \sqrt{\frac{2 q_{e} \Delta V}{m_{e}}} \times \Delta V .
$$

Although for the general case of spinning tethers the bias $\Delta V$ will be time-dependent we can here (conservatively) assume that the temperature variation follows quasi-steadily the tether attitude change, and compute the maximum temperature for the case in which the tether is aligned with the motional electric field vector $\mathbf{E}_{m}$. In such case, the maximum bias along the tether is:

$$
\Delta V_{\max }=E_{m} L,
$$

reached at the tether anodic end when $\zeta$ is set to 1 (maximum current). After plugging Eqs. $(18,19)$ into Eq.(17) we obtain the maximum temperature: 


$$
T_{\max }=\left(\frac{2 q_{e}^{3}}{\pi^{2} \sigma_{B}^{2} m_{e}}\right)^{1 / 8} \times \frac{N_{e}^{1 / 4} E_{m}^{3 / 8} L^{3 / 8}}{\varepsilon_{t}^{1 / 4}}
$$

In the worst case scenario of retrograde low altitude $\left(1.06 r_{J}\right)$ circular orbit $E_{m}$ and $N_{e}$ are about $20 \mathrm{~V} / \mathrm{m}$ and $6.5 \times 10^{9} \mathrm{~m}^{-3}$, respectively. If we take $\varepsilon_{t}=0.8$ for our aluminum tape and consider $5 \mathrm{~km}$ as our maximum tether length the temperature $T_{\max }$ barely reaches $300 \mathrm{~K}$, meaning tether heating is not an issue.

It can be shown that tether bowing, which scales as $L^{5 / 2}$ [4], is even less of an issue for the tether sizes considered in the present study.

\section{Conclusions}

In this work we considered a final-stage mission with a tethered SC starting in circular, equatorial orbit at a radius below the Jovian Radiation Belts. The tether can make its spacecraft spiral in slowly in a controlled manner while generating power onboard. The proximity to Jupiter, the in situ conditions, first at a particulate ambient and later down in the atmosphere, and the possible use of the tether itself as instrument in active experiments, offer scientific opportunities for studies on ring grains, and the interior and atmosphere of Jupiter. We discussed tether performance and design. For a selected SC mass and on board power we determined both the full deorbiting time and the tether length required.

\section{References}

[1] "Radioisotope Power Systems: An Imperative for Maintaining US Leadership in Space Exploration," Tech. rep., National Research Council (Aeronautics and Space Engineering Board, Space Studies Board, Engineering and Physical Sciences, 2009.

[2] "Jupiter Europa Orbiter and Jupiter Ganymede Orbiter Concept," last accessed: June 2009, http://opfm.jpl.nasa.gov/europajupitersystemmissionejsm/.

[3] Sanmartin, J., Lorenzini, E., and Martinez-Sanchez, M., "Electrodynamic Tether Applications-An Overview," to be published.

[4] Sanmartin, J., Lorenzini, E., Garrett, H., Bombardelli, C., and Bramanti, C., "Electrodynamic Tether at Jupiter-I: Capture Operation and Constraints," IEEE Transactions on Plasma Science, Vol. 36, No. 5, 2008, pp. 2450-2458.

[5] Sanmartin, J., Charro, M., Lorenzini, E., Garrett, E., Bombardelli, C., and Bramanti, C., "Electrodynamic Tether at Jupiter-II: Fast Moon Tour after Capture," IEEE Transactions on Plasma Science, Vol. 37, No. 4, 2009, pp. 620-626.

[6] Bombardelli, C., Lorenzini, E., and Sanmartin, J., "Jupiter Power Generation at Constant Orbital Energy," Journal of Propulsion and Power, Vol. 5, No. 2, March-April 2009, pp. 415-424.

[7] Charro, M., Sanmartin, J., Bombardelli, C., and Lorenzini, E., "Electrodynamic Tether Mission to Low Jovian Orbit," in preparation.

[8] Burns, J., Shaffer, L., Greenberg, R., and Showalter, M., "Lorentz Resonances and the Structure of the Jovian Ring," Nature, Vol. 316, July 1985, pp. 115-119.

[9] F.Bagenal, Dowling, T., and McKinnon, W., editors, Jupiter. The Planet, Satellites, and Magnetosphere, cambridge univ. press, cambridge, uk ed., 2004.

[10] Sanmartin, J., Charro, M., Peláez, J., Tinao, I., Elaskar, S., Hilgers, A., and Martinez-Sanchez, M., "Floating Bare Tether as Upper Atmosphere Probe," J. Geophysical Research, Vol. 111, No. A11310, 2006, pp. 1-15. 
[11] Divine, N. and Garrett, H., "Charged Particle Distributions in Jupiter's Magnetosphere," Journal of Geophysical Research, Vol. 88, No. A9, September 1983, pp. 6889-6903. 